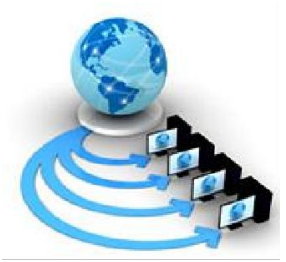

Volume 11, No. 3, May-June 2020

ISSN No. 0976-5697

International Journal of Advanced Research in Computer Science

RESEARCH PAPER

Available Online at www.ijarcs.info

\title{
REAL TIME EYE TRACKING FOR PASSWORD AUTHENTICATION
}

\author{
Dr. T R Muhibur Rahman \\ Associate Professor \\ Department of Computer Science \& Engineering \\ Ballari Institute of Technology and Management \\ Ballari, India
}

\author{
Neha K \\ $8^{\text {th }}$ Semester B.E.(CSE)
}

Ballari Institute of Technology and Management

Ballari, India

Priyanka G

$8^{\text {th }}$ semester B.E.(CSE)

Ballari Institute of Technology and Management

Ballari , India

\author{
Reshma M \\ $8^{\text {th }}$ semester B.E.(CSE) \\ Ballari Institute of Technology and Management \\ Ballari, India \\ Pavitra V \\ $8^{\text {th }}$ semester B.E.(CSE) \\ Ballari Institute of Technology and Management \\ Ballari , India
}

\begin{abstract}
The Generality of today's authentication systems, including password, fingerprint scanners and Personal Identification Number(PIN) entry, are based on one time, static authentication methods which are prone to attacks. Real time eye tracking contributes significantly for Patients who are incapacitated, internet study, gaze based password entry as well as house hold security applications. The use of real time eye tracking and password authentication system has a wider possibility for reliability, but in this case the system must be secure and restrained for Eye Detection and Tracking. For the user's security and validation (authentication) personal identification numbers are used abroad thereby reducing the chances of being attacked. In static authentication methods, since the password has to be entered physically using PIN's there are possibilities for tracking the password using shoulder surfing, in order to avoid this spy game of tracking the password, hands off gaze- based PIN entry techniques are used. The smart camera is used to detect the eye position and for Gaze-based PIN Identification thereby doing the data processing. This project is focused on a real time hands off and gaze based PIN entry, detecting the eye position and tracking the eye movement using the smart camera.
\end{abstract}

Keywords: Smart Camera, Personal Identification Number Entry, Eye Detection and Tracking, Gaze-based PIN Identification

\section{INTRODUCTION}

Personal Identification numbers are widely used for many applications. 4 digits or 6 digits PINs are used as money management in ATM (automatic teller machines). They are also used for unlocking personal devices and also while handling the electronic transactions. The fraud attack on automated teller machines has been increased. The user must enter the code in open which makes the PIN entry vulnerable to tracking of password using techniques such as shoulder surfing.

The primary point of this work is to enter the secret number utilizing look and distinguish the entered PINs utilizing a smart camera through constant eye location and following it's movement. Following the movement of the eye under various conditions including diverse situation of the face, head developments and eye obstacle including eye location is depicted by the exploratory assessment of calculation to decide the flexibility of the framework for continuous applications. National Instruments Vision developer and LabView are the product utilized for eye following and the continuous camera is utilized for recording eye focus area. The on-board information preparing and assortment is approved by the smart camera. A layer of security for Physical PIN sections are made sure about by the non-contact PIN based confirmation and are foreseen to decrease the shakiness of the verification procedure.

\section{LITERATURE REVIEW}

\section{A. Smart Eye Tracking System}

- Aniwat Juhong et al.[1] proposed a smart eye tracking system which is maily designed for elderly people and for people with disabilities.

- Architecture : 


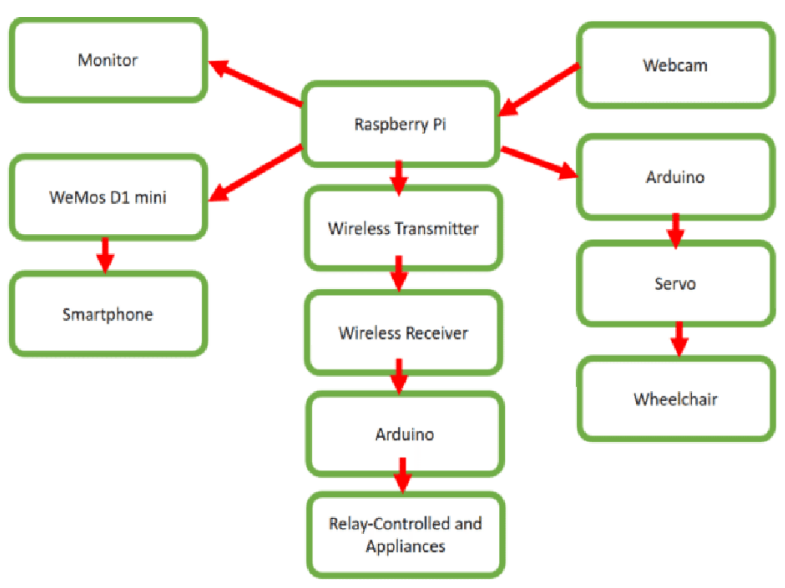

Fig 1. System overview of smart eye tracking system

- Methodology:

Smart eye tracking system uses Hough circle transformation to detect the circle, the eye blink detection is used to simulate the enter key board. To control the mouse position, the eye ball position is used.

- Limitations :

- The web cam that is used in this system is quite large in size and may bother the user.

- The system is not cost effective.

B. Quantitative analysis of tennis experts' eye movement skill

- Sota Shimizu et al.[2] proposed a system called Quantitative analysis of tennis experts' eye movement skill to measure the eye movements with respect to 2 categories i.e. with experts and beginners.

- Architecture :

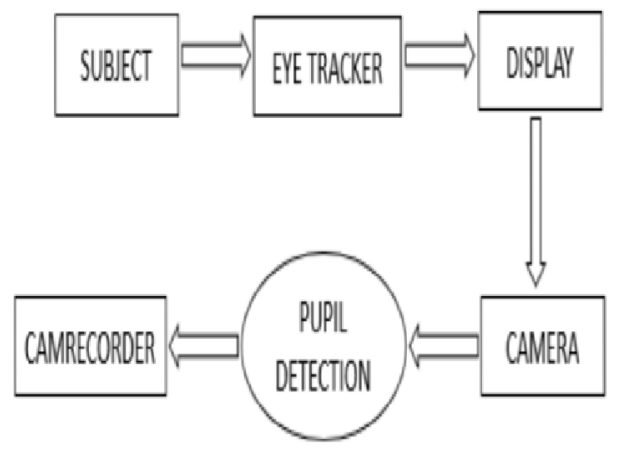

Fig 2 : System overview

- Methodology :

Quantitative Analysis of Tennis Experts, Eye Movement Skill includes eye tracking where a camera is used to calculate the subject's eye position relative to the display and also the size of the pupil. The next step includes recording and measuring the beginners' and experts' gaze data. After this , Comparison and Observation of a Timeseries of Likelihood Distribution takes place.

- Limitations:

The measurement and analysis of the subject's gazed data is not done on an actual tennis court or in real time.

\section{Eye movement-related EEG potential pattern recognition for real time $\mathrm{BMI}$}

- Takuma Ito et al.[3] proposed a system namely Eye movement-related EEG potential pattern recognition for real time BMI which aims at rapid BMI pattern recognition for the eye-ball movement.

- Architecture :

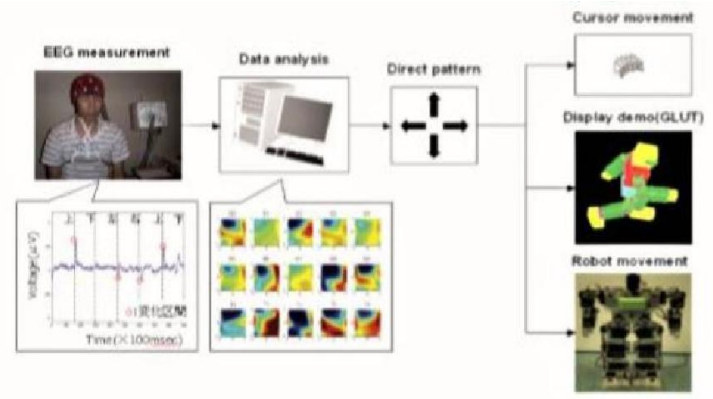

Fig 3. System overview of Eye movement-related EEG for real time BMI

- Methodology :

The first step involved in this method is the Scheme Optimization. In this step, the number of hidden layers were reduced to find the optimal scheme by eliminating the tolerant factor. The next step is the Weight Tuning, here the discrimination accuracy is improved. After weight tuning, Eye Induced Interface takes place. It includes around $500 \mathrm{~ms}$ interface which is suited for human interface .

- Limitations:

Reinforcement leaning discriminator of $\mathrm{MNN}$ scheme shows lower performance for this input patterns compared with ISE, LTNEW discriminator.

\section{REAL TIME EYE DETECTION SYSTEM}

\section{SMART CAMERA}

Eye detection to capture the eye patterns of readers based on their reading skill levels is detected by the system. Here the main components of eye tracking system include NI 1762 start camera, $12 \mathrm{~mm}$ lens computer and the Gigabit Ethernet 
Interface. A digital keypad is set for eye gazing and eye tracking, here the users are asked to read texts while on the other hand the eye location of the users are saved on board the smart camera across the image frames, the users eye can be directly viewed above the keypad screen and in this application particularly the viewer gazes at the digits of digital keypad.

The primary bit of leeway of smart camera is information preparing, catch and capacity to store. On the grounds that the camera is double processor it works with applications, for example, information lattice code, optical character acknowledgment and example coordinating. The camera is outfitted with $1 / 3$ inch sony $1 \mathrm{CX} 424$ AL strong state picture sensor. This framework or system in entire forms high goals dim scale pictures which is essentially required for the execution or usage of continuous eye movement calculation. The information can be moved to different gadgets through FTP or Ethernet equipment which is associated with smart camera. As the client stops over every digit for a few seconds the camera outline rate determination is firm for this application. Around seven edges for every second can be prepared by the eye identification calculation. For look based secret phrase recognizable proof more slow cameras can likewise be utilized.

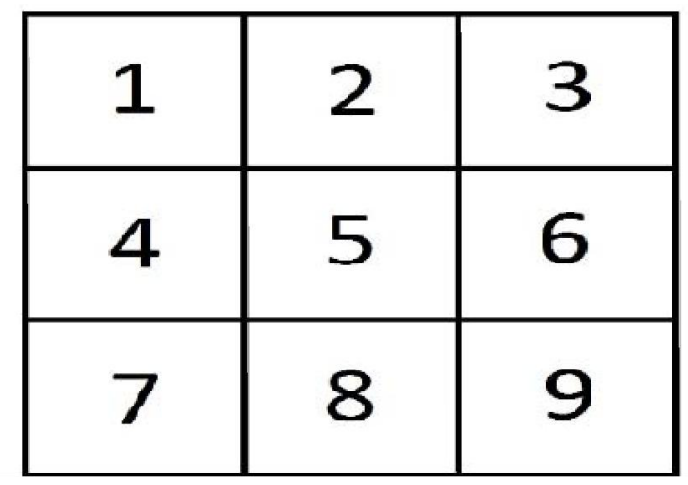

Fig 4. Digital keypad for eye gazing and tracking

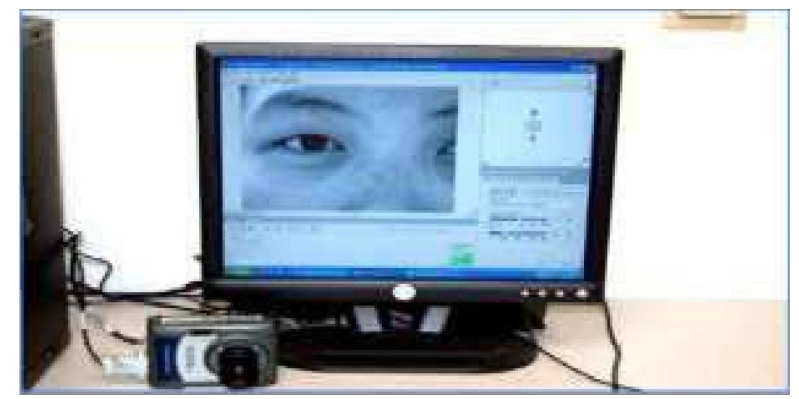

Fig.5. NI Smart camera and Eye tracking system

\section{ALGORITHM TO LOCATE THE EYE AND IT'S MOVEMENT.}

The eye discovery calculation will begin by catching the pictures of clients face and it recognizes the situation of the eye., via preparing the calculation a solitary edge of the client's eye is caught and is spared as the eye layout. A similar play of activity is trailed by the eye movement calculation.

It catches the picture of the client's face and identifies the situation of the eye. The spared layout is utilized for eye coordinating and the continuous picture outline for best fit is examined utilizing standardized cross-connection. The transformation of the co-ordinate framework is done to normalize the co-ordinates of the eye area that is accounted for and furthermore to catch the pictures of the eyes during the various developments of the head like the inclining of the head. Utilizing circle or oval coordinating the iris of eye is identified from the best coordinated eye picture through edge identification and Hough changes. The centre is processed scientifically as the focal point of the oval and is recorded on a spreadsheet on board camera as the eye place. The LabView programming is utilized for eye location and following it's movement.

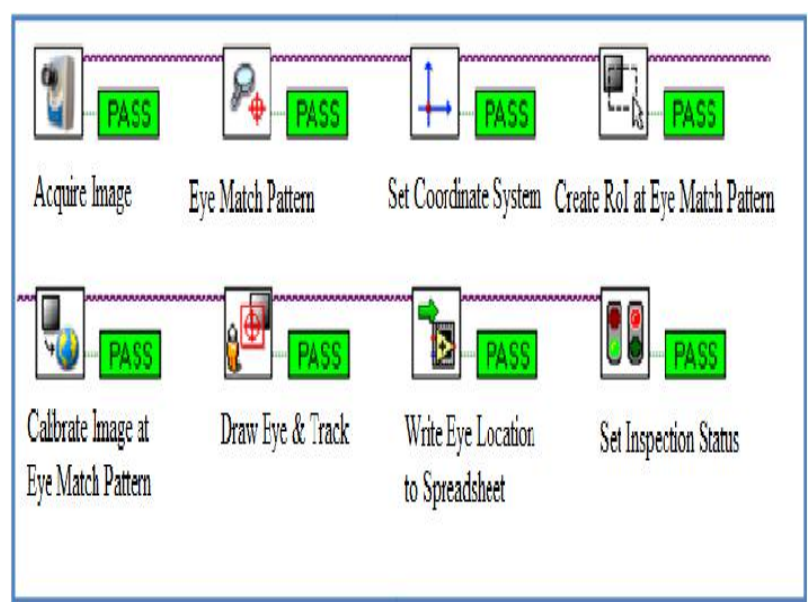

Fig 6. LabView square chart for eye discovery and following it's movement.

\section{ALGORITHM TO FOLLOW THE MOVEMENT OF THE EYE.}

Here the eye movement can be followed constantly as the camera is modified and can be halted just when the client needs to.

Figure 7 is the flow chart of the calculation of Real time eye movement. 


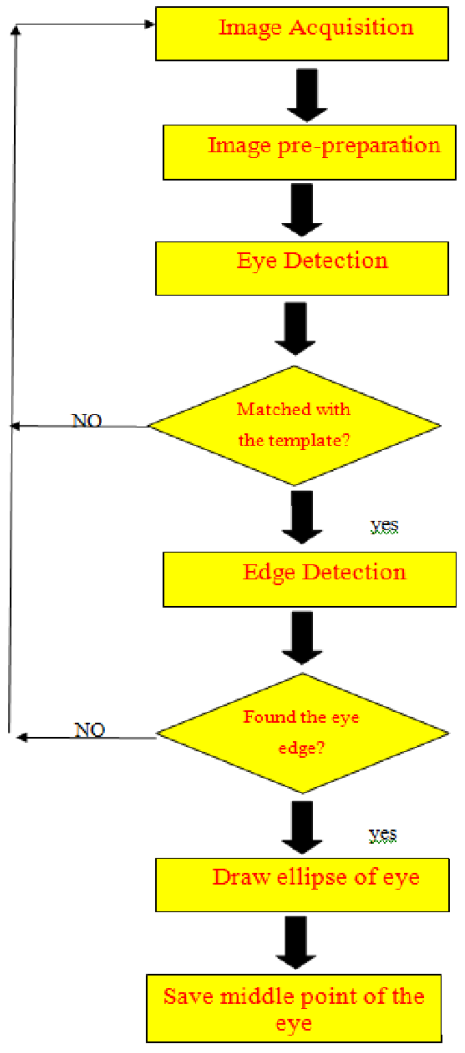

Fig 7. Flowchart of Algorithm to track the real time eye movement.

Step 1 - Image Acquisition: Smart camera is utilized to consequently secure the picture.

Step 2 - Image Pre-preparing: The keen camera is utilized again to change over the gained crude pictures into dark scale pictures.

Step 3 - Eye Detection: Using the format coordinating method, the client's eye is identified. In layout coordinating, a format is contrasted and the picture obtained utilizing a coordinating measurement. The coordinating measurement gives a proportion of closeness between the two formats. This closeness is changed over into a numerical worth .

Step 4 - Edge Detection: If the eye is identified, new Region of intrigue (ROI) which covers just the eye is separated to diminish the handling region. An edge recognition strategy is applied to the new ROI to discover focuses around the oval or hover of the eye.

Step 5 - If at any rate 3 focuses (for circle) or 4 focuses (for oval) are discovered, the circle or the oval of the eye will be drawn. Something else, the present casing is skirted with no match.

Step 6 - If the oval/hover of the eye is drawn the directions of the focal point of determined in the camera's processor and spared to spreadsheet for future reference. These directions are figured as the focal point of the square shape bouncing the distinguished eye.

\section{PERSONAL IDENTIFICATION NUMBER ENTRY}

User enters the PIN by looking at the digits on the keypad, here the user observes the digits closely for a few seconds with his/her eyes and subsequently moves to the next digit on the PIN pad, as the user is viewing the digits on the keypad the partial face of the user is captured by the smart camera in sequential frames.

Later the centre of the eye is located with the help of the processing algorithms such as Eye detection algorithm and the Cartesian coordinates are recorded which represents the centre of the eye. As soon as the PIN entry is completed the eye tracking application stops.

\section{GAZE BASED PIN IDENTIFICATION}

The eye place arrangements are on a level plane and vertically plotted on the 2D spread sheet for PIN ID. Bunch investigation or bunching is an assignment of collection a lot of items where the items in same gathering are more like each other than that of different gatherings and this idea of grouping is utilized to assemble the information focuses. The sequence in which the digits are looked won't be resolved rather the looked digits will be resolved, so as to show the request for looked digits a $3 \mathrm{D}$ associated diagram is plotted and along these lines the request for entered digits is resolved.

\section{CONCLUSION}

Results from user's study show that eye tracker gives a good performance for user's authentication via iris recognition. The eye tracker has become widely available for user interface devices, perhaps replacing traditional offers they might offer the additional benefit of real time, continuous user authentication. For gaze- based PIN identification a smart -camera based eye tracking system is integrated into new application.

\section{FUTURE ENHANCEMENT}

The system has been successfully tested with a nine digit keypad and can further be extended to a combination of characters and digits .

Future work includes incorporating the eye tracking algorithm into the real-time applications for all in one password authentication system. Adding to this, the hands off gaze based password entry and its authentication can be extended to mobile devices, computers and other devices which incorporates a camera within it. 


\section{REFERENCES}

[1] Aniwat Juhong, T. Treebupachatsakul and C. Pintavirooj, "Smart Eye Tracking System" Research Gate, January 2018.

[2] Sota Shimizu, Takumi Kadogawa, Shu-ichi Kikuchi, Takumi Hashizume, "Quantitative analysis of tennis experts' eye movement skill" IEEE 13th International Workshop on Advanced Motion Control (AMC), IEEE, INSPEC Accession Number: 14349640 02-June-2014.

[3] Takuma Ito, Tomoyuki Shinji, Hideyasu Sumiya, Mituru Baba, "Eye movement-related EEG potential pattern recognition for real-time BMI", Proceedings Of SICE Annual Conference, IEEE, INSPEC Accession Number: 11594913, 14-October-2010

[4] R Revathy and R Bama, "Advanced Safe PIN Entry Against Human Shoulder Surfing" IOSR Journal of Computer Engineering, vol 17, issue 4, ver. II , pp 9-15 , July-August 2015.
[5] J. Weaver, K. Mock and B. Hoanca, "Gaze-Based Password Authentication through Automatic Clustering of Gaze Points", Proc 2011 IEEE conf. on Systems, Man and Cybernetics , 2011.

[6] "ATM Fraud, ATM Black Box Attacks Spread across Europe", European ATM Security Team (E.A.S.T), online, posted on 11 April 2007.

[7] K. Mowery, S. Meikle john and S. Savage, "Heat of the Moment: Characterizing the Efficacy of Thermal Camera-Based Attacks," WOOT' vol 11, pp 1-8, August 2011.

[8] M. Mehrubeoglu, E. Ortlieb, L. McLauchlan, L. M. Pham, "Capturing reading patterns through a real-time smart camera iris tracking system," Proc. SPIE, vol. 8437, id. 843705, 2012.

[9] M. Mehrubeoglu, H. T. Bui and L. McLauchlan, "Realtime iris tracking with a smart camera," Proc. SPIE 7871, 787104, 2011.

[10] Smart Cameras for Embedded Machine Vision, (product information) National Instruments. 